\title{
Case study of a complex active-region filament eruption
}

\author{
X. L. Yan ${ }^{1,2}$, Z. Q. Qu ${ }^{1}$, D. F. Kong ${ }^{1,3}$, L. H. Deng ${ }^{1,3}$, and Z. K. Xue ${ }^{1,3}$ \\ 1 Yunnan Observatories, Chinese Academy of Sciences, 650011 Kunming, PR China \\ e-mail: yanxl@ynao.ac.cn \\ 2 Key Laboratory of Solar Activity, National Astronomical Observatories, Chinese Academy of Sciences, 100012 Beijing, PR China \\ ${ }^{3}$ University of Chinese Academy of Sciences, Zhongguancun, 100049 Beijing, PR China \\ Received 14 February 2012 / Accepted 6 July 2013
}

\section{ABSTRACT}

\begin{abstract}
Context. We investigated a solar active-region filament eruption associated with a C6.6 class flare and a coronal mass ejection (CME) in NOAA active region 08858 on 2000 February 9.

Aims. We aim to better understand the relationship between filament eruptions and the associated flares and CMEs.

Methods. Using BBSO, SOHO/EIT, and TRACE observational data, we analyzed the process of the active-region filament eruption in the chromosphere and the corona. Using the SOHO/MDI magnetograms, we investigated the change of the magnetic fields in the photosphere. Using the GOES soft X-ray flux and the SOHO/LASCO images, we identified the flare and CME, which were associated with this active-region filament eruption.

Results. The brightenings in the chromosphere are a precursor of the filament expansion. The eruption itself can be divided into four phases: In the initial phase, the intertwined bright and dark strands of the filament expand. Then, the bright strands are divided into three parts with different expansion velocity. Next, the erupting filament-carrying flux rope expands rapidly and combines with the lower part of the expanding bright strands. Finally, the filament erupts accompanied by other dark strands overlying the filament.The overlying magnetic loops and the expansion of the filament strands can change the direction of the eruption.

Conclusions. The time delay between the velocity peaks of the filament and that of the two parts of the bright strands clearly demonstrates that the breakup of the bright loops tying on the filament into individual strands is important for its eruption. The eruption is a collection of multiple processes that are physically coupled rather than a single process.
\end{abstract}

Key words. Sun: activity - Sun: filaments, prominences - Sun: flares - Sun: coronal mass ejections (CMEs) - Sun: surface magnetism

\section{Introduction}

Investigating the triggering mechanism of filament eruption is a prominent issue in current solar physics research. It is widely accepted that flares, filament eruptions, and coronal mass ejections (CMEs) are different aspects of the same physical process, including plasma ejection and magnetic energy release (Shibata et al. 1995; Forbes 2000).

Filaments are believed to consist of cool gas supported in the corona by large-scale curved and twisted magnetic flux tubes (e.g. Priest et al. 1989; Rust \& Kumar 1994; Amari et al. 1999; Gibson \& Fan 2006). Van Ballegooijen \& Martens (1989) and Aulanier et al. (2010) proposed that shearing and converging footpoint motions will force magnetic reconnection between the arcade field lines, progressively transforming the arcade magnetic field lines into a twisted flux tube. The importance of the filament channel as a precondition for filament formation has been stressed by the observations of Gaizauskas (1998) and other researchers. Moreover, the convergence of opposite polarity magnetic field is a necessary condition for the formation of filaments (for a review see Martin 1998).

Up to now, there is no conclusive answer for what triggers the filament eruption. The models that are used to interpret the initiation of filament eruptions have been proposed in the lastest decades as follows:

(1) The catastrophe model. Forbes (1990) and Forbes \& Isenberg (1991) used a two-dimensional numerical simulation to exhibit that the loss of equilibrium of the flux rope may be the main reason for the filament eruption. They confirmed that the stable configuration containing the filament loses equilibrium when the filament current exceeds a critical value. When the equilibrium is lost, the filament is magnetically driven upward. The loss of equilibrium leads to the sudden formation of a current sheet, and if a rapid reconnection occurs in this sheet, the flux rope can escape from the Sun and evolves into a CME (Lin et al. 1998; Lin \& Forbes 2000; Priest \& Forbes 2002).

(2) The tether-cutting model. This model suggests that selfamplifying magnetic reconnection occurs in the core of the sheared magnetic arcade loops and transforms part of the reconnected field lines into a twisted flux rope, which is able to erupt as a result of the cut tethers. Prior to the eruption, the field lines in the strongly sheared core appearing as $\mathrm{S}$ or reverse-S shape are aligned along the polarity inversion line in the middle of opposite polarities (Moore \& LaBonte 1980). Moore et al. (2001) presented six events to support this model by using the soft X-ray images from Yohkoh and magnetograms from Kitt Peak National Solar Observatory.

(3) The magnetic breakout model. Antiochos et al. (1999) did numerical simulations to demonstrate that the reconnection removes the unsheared fields above the sheared core flux near the neutral line and thereby, allows the core flux to burst. Lynch et al. (2004) and DeVore \& Antiochos (2008) have shown this model through magnetohydrodynamic simulations. The observations of Gary \& Moore (2004), Shen et al. (2012), and Yan et al. (2012) also provided the positive evidence of this model.

(4) The kink instability model. Hood \& Priest (1979) have investigated the magnetohydrodynamic stability of a coronal magnetic loop to kink perturbations. When a magnetic loop 
is twisted beyond a critical value, it becomes kink unstable. The magnetic loop equilibrium presented by Titov \& Démoulin (1999) is kink-unstable for twists $\Phi>\Phi_{\mathrm{c}}$ with $\Phi_{\mathrm{c}} \approx 3.5 \pi$ at a loop aspect ratio $R / a \approx 5$. Using the flux rope model of Titov \& Démoulin (1999) as the initial condition in MHD simulations, Török \& Kliem (2005) confirmed that the helical kink instability of a twisted magnetic flux rope can be the mechanism of the initial driver of solar eruptions. Fan (2005) performed MHD simulations to show that the nonlinear evolution of the kink instability facilitates the loss of confinement of the flux rope by changing its orientation at the apex, such that it becomes easier for the flux rope to pass and erupt through the arcades. The idea of sigmoids being part of a kinked flux rope was supported by estimating the deformation of the kinked rope, which gave values consistent with the range observed for sigmoids (Török et al. 2004). Observations of filament eruptions strongly suggested that a helical kink in flux rope topology was present (Ji et al. 2003; Rust \& LaBonte 2005; Williams et al. 2005; Alexander et al. 2006). Zhou et al. (2006) described a clear case of two successive coronal mass ejections driven by the kink instability of an eruptive prominence. In addition, Srivastava et al. (2010) also showed that the kink instability of the twisted magnetic loop triggered a B5.0 class solar flare.

(5) The flux cancellation and emerging flux triggering mechanism. Chen \& Shibata (2000) and Lin et al. (2001) proposed the emerging flux triggering mechanism for filament eruptions and CMEs, motivated by the observed correlation among reconnection-favored emerging flux, filament eruption, and CMEs (e.g., Feynman \& Martin 1995; Wang \& Sheeley 1999; Jing et al. 2004; Jiang et al. 2007; Yan et al. 2011). Linker et al. (2003) carried out a simulation and found that flux cancellation can form a stable flux rope configuration within the helmet streamer. With continued flux cancellation, the helmet streamer is destabilized, and the flux rope begins to erupt.

The models 1, 2, and 3 are all required for the occurrence of magnetic reconnection. However, the evidence of magnetic reconnection is very difficult to find from observations. The model 2 requires the tether cutting of the magnetic field lines and forms a flux rope. The models 4 and 5 are relatively easy to be determined from observation. In this event, there was not obvious flux emerging and cancellation observed from the magnetograms before the filament eruption. Also, the reconnection of the overlying unsheared field was not observed. The brightenings in the TRACE $1600 \AA$ image were found before the expanding of the filament. This phenomenon could be an indication of tether cutting. Due to the lack of the $\mathrm{H} \alpha$ data, we cannot give a conclusion. During the filament expansion, the deformation of the filament has also been observed. Compared with the models, the filament system in this event contained several different magnetic structures (bright strands and dark strands) and not a single flux rope, which intertwined together. The expansion of the bright strands of the erupting filament-carrying flux rope is prior to the filament eruption. In this event, we focus on the change of the strands of the filament during the filament eruption, which has not been concerned by these models mentioned above.

\section{Observations}

An active-region filament eruption associated with a C-class flare and a CME in NOAA AR 08858 was observed by Big Bear Solar Observatory (BBSO; Steinegger et al. 2000),

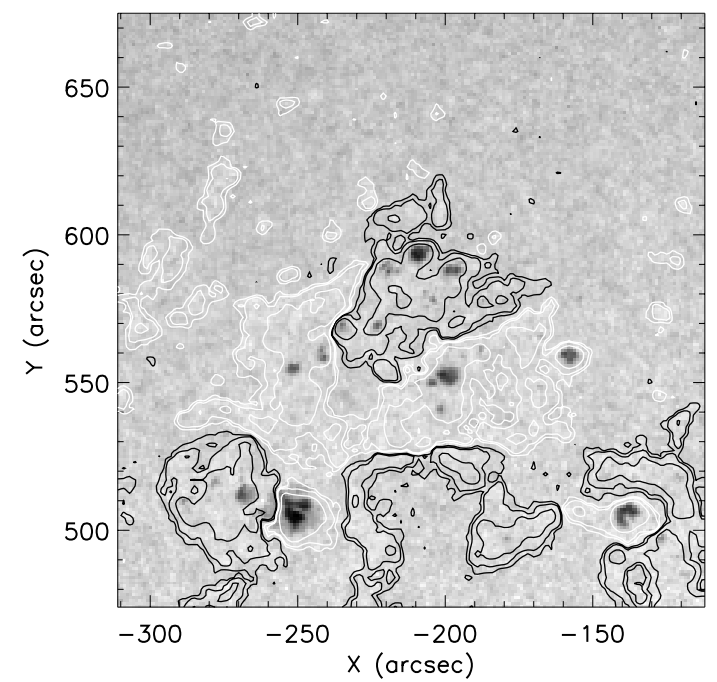

Fig. 1. Alignment of the TRACE white-light image at 00:15:52 UT on February 9 and the SOHO/MDI magnetogram at 23:59:30 UT on February 8. The levels of the contours of the magnetic fiels are $\pm 50 \mathrm{G}$, $\pm 100 \mathrm{G}$, and $\pm 300 \mathrm{G}$.

Transition Region and Coronal Explorer (TRACE; Handy et al. 1999), Extreme ultraviolet Imaging Telescope (EIT; Delaboudinière et al. 1995), Michelson Doppler Imager (MDI; Scherrer et al. 1995) and Large Angle and Spectrometric Coronagraph (LASCO; Brueckner et al. 1995) on board Solar and Heliospheric Observatory (SOHO; Domingo et al. 1995), and Geostationary Operational Environmental Satellite (GOES). A BBSO $\mathrm{H} \alpha$ image and an SOHO/EIT $304 \AA$ image are used to identify the existence of the filament in this active region. The TRACE $1600 \AA$ images were used to investigate the change of this active region in the chromosphere. The observation duration of TRACE acquired at $1600 \AA$ was from 00:42:33 UT to 01:00:09 UT. Unfortunately, there was a data gap from 00:47:33 UT to 01:00:09 UT, but the period from 00:42:33 UT to 00:47:33 UT included the most time of the filament eruption. The corresponding TRACE $171 \AA$ observations have covered the whole process of this event. Full-disk line-of-sight magnetograms from SOHO/MDI are used to show the magnetic fields in the photosphere, and TRACE $171 \AA$ images are used to indicate the topology of magnetic field lines in the corona. A CME followed by the eruption of the filament was observed by LASCO C2 on board SOHO. The data of TRACE $1600 \AA$ and the Fe IX/X $171 \AA$ images were acquired with a cadence of about $30 \mathrm{~s}-1 \mathrm{~min}$ and a pixel size of 0.5 . Full-disk line-of-sight magnetograms are taken by the Michelson Doppler Imager (MDI) on board SOHO with a 96-min cadence and a spatial resolution of $2^{\prime \prime}$ per pixel.

The images observed by TRACE are part of the Sun, while the SOHO/MDI magnetograms are full disk images. We needed to align the TRACE images and SOHO/MID magnetograms. First, we aligned the TRACE white-light image with SOHO/MDI magnetogram. According to the information from the head of the images, we can get the heliographic coordinates by using the software in the SSW. As the sunspots can be seen clear in the white-light image and the magnetogram, we can co-align accurately these images. Second, we co-aligned the TRACE white-light image and the $1600 \AA$ image according to the coordinate of the TRACE images. Third, we co-aligned the TRACE $1600 \AA$ image and the SOHO/MDI magnetograms. Figure 1 shows the alignment of the TRACE white-light image 


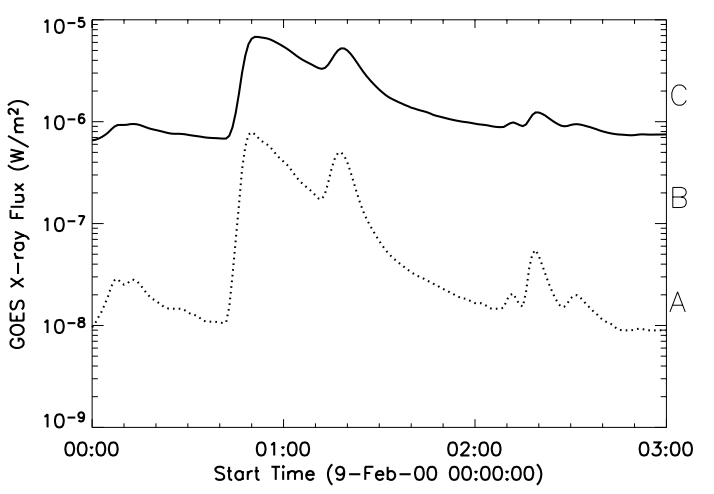

Fig. 2. Evolution of GOES soft X-ray emission (solid line: 1-8 $\AA$; dashed line: 0.5-4 A) for the C6.6 flare on February 9, 2000.

at 00:15:52 UT on February 9 and the SOHO/MDI magnetogram at 23:59:30 UT on February 8. The levels of the contours of the magnetic fiels are $\pm 50 \mathrm{G}, \pm 100 \mathrm{G}$, and $\pm 300 \mathrm{G}$.

\section{Process of the filament eruption}

\subsection{EUV continuum observations}

Figure 2 shows the evolution of GOES 10 soft X-ray emission flux (Solid line: $1-8 \AA$; Dashed line: $0.5-4 \AA$ ) for a C6.6 flare on February 9, 2000. According to the GOES 10 observation, the C6.6 flare started at 00:42 UT, peaked at 00:51 UT, and ended at 01:09 UT.

Figure 3 shows the $\mathrm{H} \alpha$ image observed by BBSO on 2000 February 7 , the $\mathrm{H} \alpha$ image observed by BBSO at 19:33:42 UT with the SOHO/MDI magnetograms at 19:11:30 UT superimposed on 2000 February 7, and the $304 \AA$ image observed by SOHO/EIT on 2000 February 8 . The white arrows indicate the filament, while the black arrow indicates the bright loop. The filament exhibited a slender long shape. The filament was not seen clearly from Fig. 3c, because the filament was along the bright loop whose brightening covered the filament. The bright loop was named as F1 and the filament was named as F2 in Fig. 5.

The production of the bright patches of the flare can be seen from the TRACE $1600 \AA$ images on 2000 February 9 (see from Fig. 4). The bright patches 1 and 2, marked by the white circles in Fig. 4a, first appeared. After thirty seconds, the bright patches 3 and 4, marked by the white circles in Fig. 4b, appeared. The four bright patches are marked in Figs. $4 b-4 d$ by the white circles $1,2,3$, and 4 . The intensities of these bright patches increased from 00:42:33 UT to 00:44:03 UT. In this period, the filament cannot be seen clearly from the TRACE $1600 \AA$ images. At 00:44:33 UT, the intensities of the four bright patches strengthened a little. At the same time, the topology of the filament marked by the white box can be seen clearly in Fig. 4f. From 00:45:03 UT to 00:47:33 UT, the filament gradually expanded upward. The white boxes indicate the positions of the filament with time. Moreover, the brightenings of the patches 1 and 2 were enhanced and the areas became larger, while the intensities of the other patches signed by circles 3 and 4 gradually increased. In contrast, the bright patches 2, 3 and 4 were found to be around the filament. It is noteworthy that the appearance of chromospheric brightening was ahead of the expansion of the filament. In other words, the onset of the C6.6 flare was earlier than the filament expansion. The time difference was about $3 \mathrm{~min}$. Two bright ribbons appeared at the final phase of the C6.6 flare (see from Fig. 41). At 01:00:09 UT, the bright patch 1 became a long narrow bright band and the bright patches 2 and 4 became a tadpole-shaped bright band. The configuration of the two bright bands consisted of the two flare ribbons marked by two green arrows in Fig. 4l. The filament drained of the bright material of bright patch 3 . The white arrows indicate the positions of the bright material that was drained by the filament. From 00:45:33 UT to 00:47:33 UT, the bright material of the patch 3 was gradually drained as the elevated filament material (see from Figs. $4 \mathrm{f}-41$ ).

\subsection{Description of coronal emission during eruption}

The eruption process of the filament in TRACE $171 \AA$ channel can be seen in Figs. 5 and 6 . The white boxes indicate the positions of the filament with time. The position of the white boxes in Figs. $5 \mathrm{a}$ and $5 \mathrm{~b}$ is the same as those boxes in Figs. $4 \mathrm{f}-4 \mathrm{i}$. The position of the white boxes in Fig. $5 \mathrm{c}$ is the same as those boxes in Figs. 4j and 4k. From 00:45:55 UT to 00:46:28 UT, there were some arcade-shaped magnetic loops over the filament marked by the white arrows and the intertwined bright and dark strands of the filament marked by F1 and F2 in Figs. 5a and 5b. The arcade-shaped magnetic loops surrounded the filament. The strands of the filament alignment were almost parallel to the plane of the arcade-shaped magnetic loops from 00:45:55 UT to 00:46:28 UT. At 00:47:23 UT, the bright and dark strands of the filament began to rotate counter-clockwise. We interpret the rotation of the filament strands during the filament expansion as owing to the presence of the arcade-shaped magnetic loops. From 00:47:23 UT to 00:47:45 UT, the strands of the filament expansion can be clearly seen (see from Figs. $5 \mathrm{c}$ and $5 \mathrm{~d}$ ). At 00:48:40 UT, the bright strands were divided into two parts marked by $\mathrm{P} 1$ and $\mathrm{P} 2$ in Fig. 5e. The upper part (P2) also departed into two parts marked by P3 and P4 at 00:49:36 UT (see from Fig. 5f). The two parts (P3 and P4) began to expand faster than $\mathrm{P} 1$, and the right part (P4) expanded slower than the left one (P3) (see from Fig. 5g). At 00:50:14 UT, the main filament and the other dark strands appeared, marked by F3 and F4. Following the expansion of the strands (P1, P3, and P4), the main filament (F3) expanded rapidly and combined with the lower part (P1) at 00:52:47 UT. After they combined, we signed the combination as P5. From 00:50:14 UT to 00:53:16 UT, the left part of the bright strands (P3) rotated clockwise. However, the main filament (F3) rotated counterclockwise from 00:50:57 UT to $00: 53: 16$ UT. Finally, the main filament alignment was almost perpendicular to the plane of the line of sight. We interpret the expansion of the main filament as owing to the expansion of the left part of the strands (P3) that dragged the main filament (F3). From 00:52:04 UT to 00:53:16 UT, the filament expanded toward the northeast of the bright strands P3 and P4, while the bright strands (P3 and P4) began to lean sideways during the rising of the filament (P5)(see from Figs. 5j, 5i). Meanwhile, the other dark strands (F4) were lifted by the main filament expansion.

At 00:54:49 UT, the dark strands (F2) began to move from east to west (see the arrows from F2 in Figs. 6a-6g) and were wound around one leg of the main filament-carrying flux rope. The profile of the erupting filament-carrying flux rope can be seen from Fig. 6d, marked by the dotted line. From Figs. 6d-6f, one can see that F2 and F3 were the different parts of the eruption filament-carrying flux rope. The dark strands (F4) overlying the erupting filament are outlined by a dashed line. Following the eruption of the filament, the dark strands (F4) also erupted. The bright strands (P3 and P4) slowly got out of the filament and fell down to the solar surface. 

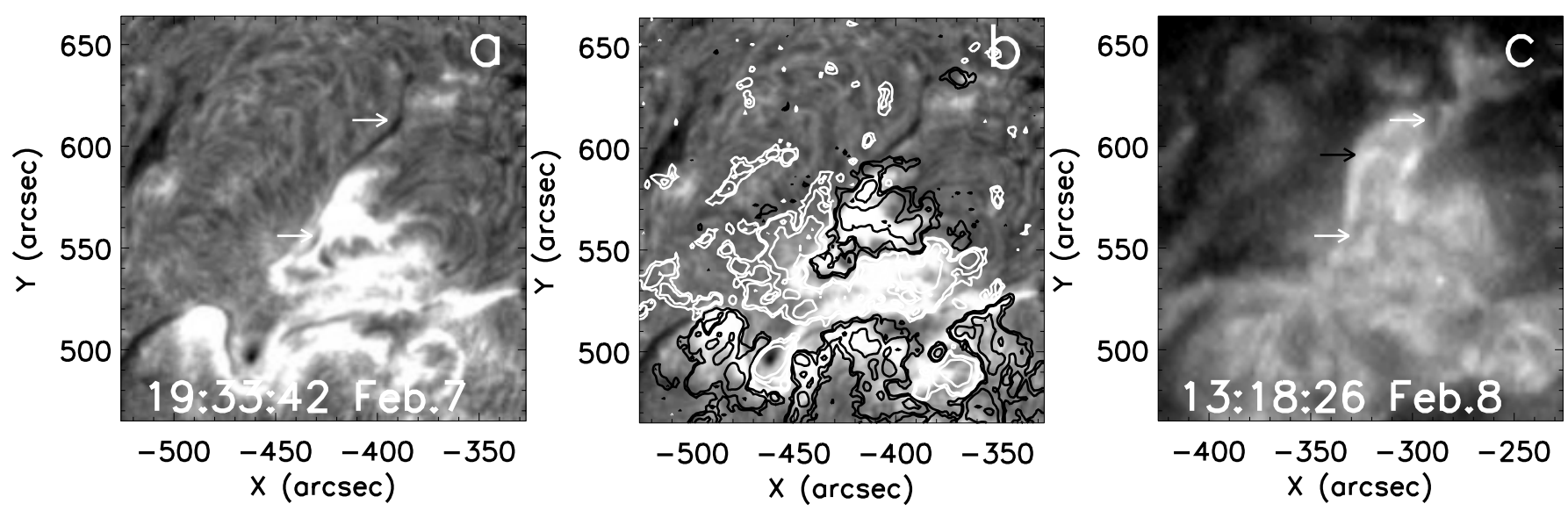

Fig. 3. a) $\mathrm{H} \alpha$ image observed by BBSO at 19:33:42 UT on 2000 February 7. b) The H $\alpha$ image observed by BBSO at 19:33:42 UT with the SOHO/MDI magnetograms at 19:11:30 UT superimposed on 2000 February 7. c) The 304 A image observed by SOHO/EIT on 2000 February 8. The white arrows indicate the filament, while the black arrow indicates the bright loop.

\subsection{Kinematics of the eruption}

The lines in Fig. 7 indicate the height (upper panel), the velocity (lower panel) of the filament bright strands (P1) (red line), the left part of the bright strands (P3) (blue line), and the main filament (F3) (green line) as a function of time, as measured from $171 \AA$ images along the dashed lines of Fig. 5 . Note that the images observed by TRACE are endured by the projection effect. It is hard to reconstruct the real shape of the loops by using single spacecraft observations. Our observations are only based on the evolution of the magnetic topology from two-dimensional data. The measured height and velocity are the projected height and velocity. We adopted the average values of three repeated measurements of the height. The filament velocity is the derivative of the height curve. The two parts of the bright strands (P1 and P2) had the same height and velocity from 00:45:55 UT to 00:48:14 UT. At 00:48:40 UT, the bright strands (P2) began to expand faster than the strands (P1). After about one minute, the bright strands (P2) departed into two parts (P3 and P4). Moreover, the expanding speed of the left part (P3) of the bright strands $(\mathrm{P} 2)$ was faster than that of the right part $(\mathrm{P} 4)$ and the bright strands (P1). The main filament (F3) began to expand at 00:50:14 UT. At 00:52:47 UT, the main filament caught up with the bright strands (P1), and then they had the same height and velocity. After the left part (P3) of the strands (P2) departed from the bright strands (P1), there were two peaks in the velocity curve, which is the same as the velocity curve of the bright strands $(\mathrm{P} 1)$, and the main filament $(\mathrm{F} 3)$. The appearance of the first peak in the bright strands (P3) velocity curve was 17 and $99 \mathrm{~s}$ earlier than the appearances of the first peak in the bright strands (P1) and the main filament (F3) velocity curve. The appearance of the second peak in the bright strand (P3) velocity curve was 26 and 93 seconds earlier than the appearances of the second peak in the bright strand (P1) and the main filament (F3) velocity curve. Note that the values of the time delay between the velocity peaks of the bright strand (P3), the main filament $(\mathrm{F} 3)$, and the bright strand $(\mathrm{P} 1)$ might be affected by the limitation of the time resolution of the TRACE data. It is very interesting that the two peaks in the bright strands (P1) and the main filament (F3) velocity curve were followed by the two peaks in the bright strand (P3) velocity curve. It is shown that the velocity of the bright strand (P1) and the main filament (F3) was very closely related to the velocity of the bright strand (P3). The velocity peaks of the bright strand (P1) delayed that of the bright strand (P3), and the velocity peaks of the main filament (F3) delayed that of the bright strand (P1). There is a clear linkage between them.

\subsection{Relation to magnetic field}

To investigate the evolution of magnetic fields, a sequence of SOHO/MDI magnetograms from 20:47:30 UT on Feb. 8 to 01:39:30 UT on Feb. 9 are presented in Fig. 8. The white and the black patches denote the positive polarity and the negative polarity, respectively. The levels of the contours of the magnetic fiels are $\pm 50 \mathrm{G}, \pm 100 \mathrm{G}$, and $\pm 300 \mathrm{G}$. The red lines denote the polarity inversion lines (PILs). From the magnetograms, there is no obvious magnetic emergence and cancellation.

To identify the locations of the brightenings, the SOHO/MDI magnetograms at 23:59:30 UT on Feb. 8 are superimposed on the TRACE $1600 \AA$ images (see from Fig. 9). The levels of the magnetic field contours are $\pm 50 \mathrm{G}, \pm 100 \mathrm{G}$, and $\pm 300 \mathrm{G}$. The green lines denote the polarity inversion lines. Two blue arrows in Fig. 9c indicate the two flare ribbons. From Fig. 9, the bright patches, marked by circles $1,2,3$, and 4 are situated near the polarity inversion line (PIL). The bright patches 2 and 4 are located in the negative polarity flux, and the bright patch 1 is located in the positive polarity flux (see from Figs. 9a and 9b). One of the C6.6 two ribbons is located in the negative polarity flux, and the other is situated in the positive polarity flux (see from Fig. 9c). The bright patch 3 is located in very weak magnetic flux and did not form as a part of the flare ribbons. Following the expansion of the filament, the bright materials began to rise along the filament (see Figs. 4f-4k). Consequently, the bright patch 3 may be the elevated filament material, which is not a part of the flare ribbons.

\subsection{Large-scale eruption}

Figure 10 shows the running difference images observed by the SOHO/LASCO on Feb. 9. At 01:31 UT, the SOHO/LASCO observed a CME, followed by the erupting filament. A bright front of CME marked in Fig. 10a first appeared. The top of the second bright part of CME exhibited an arch-shaped structure (see from Figs. 10b and 10c). Next, there was some indication for a twisted structure that would most likely be linked to the event that we discussed in this study (seen from Figs. 10d-10f). The 
X. L. Yan et al.: Case study of a complex active-region filament eruption

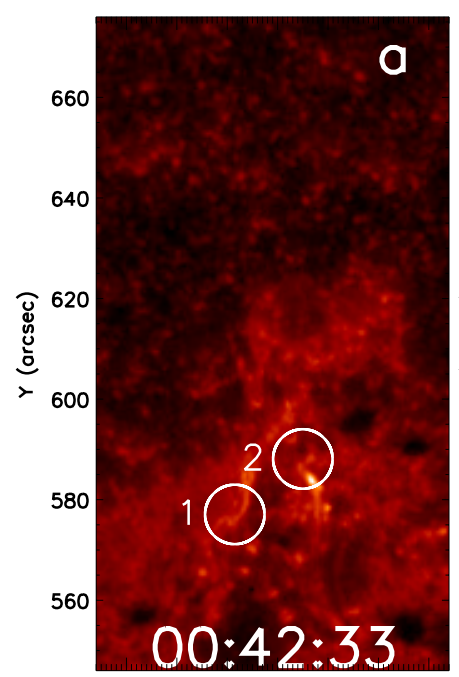

$-250-240-230-220-210-200-190$ $x$ (arcsec)
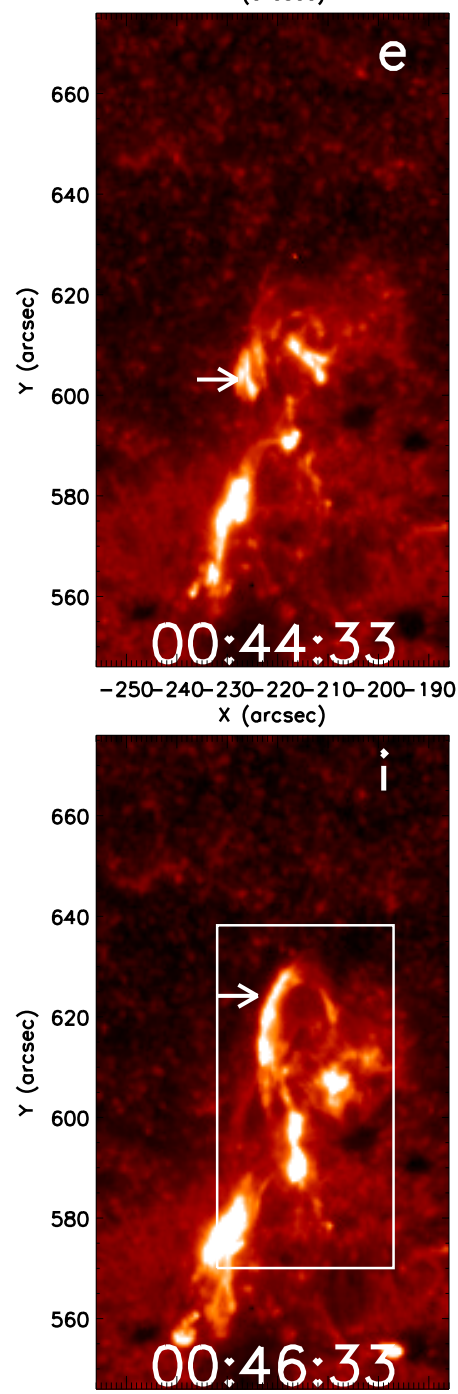

$-250-240-230$ $x$ (arcsec)

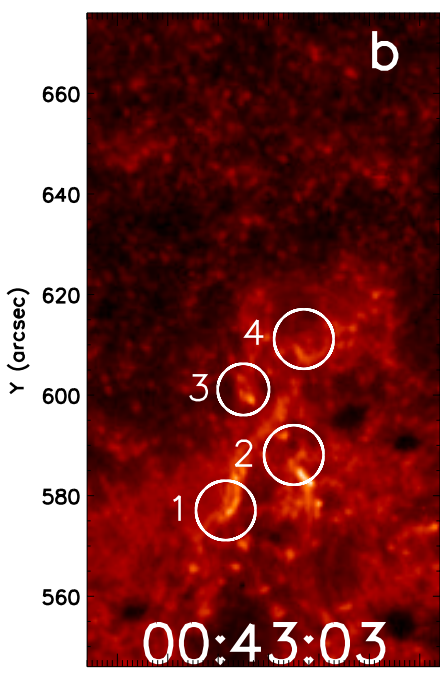

$-250-240-230-220-210-200-190$ $x$ (arcsec)
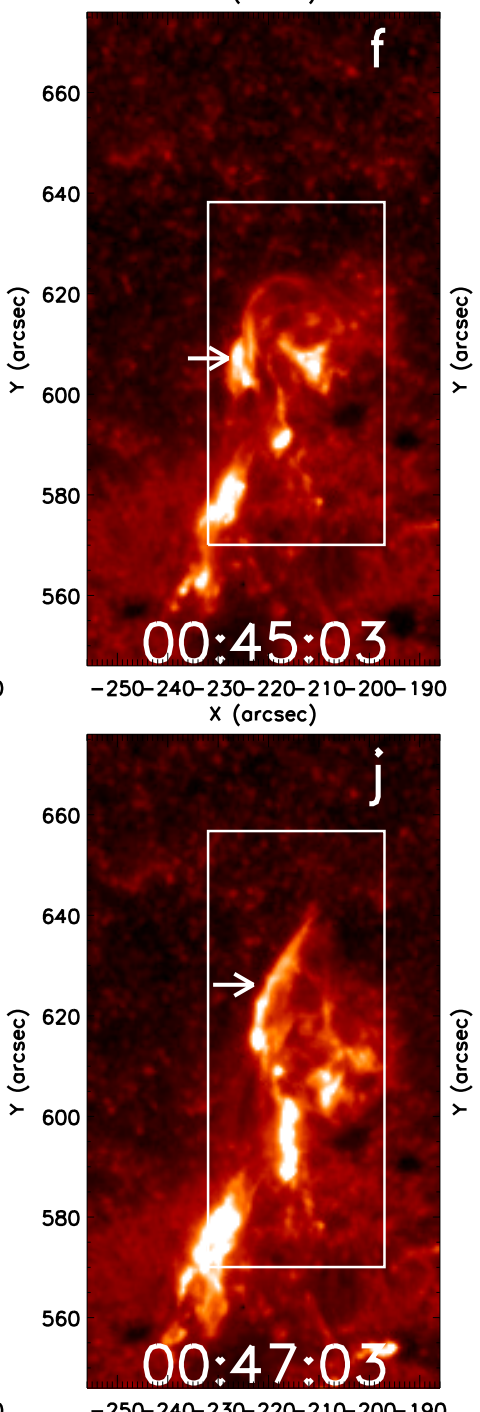

$x(\operatorname{arcsec})$

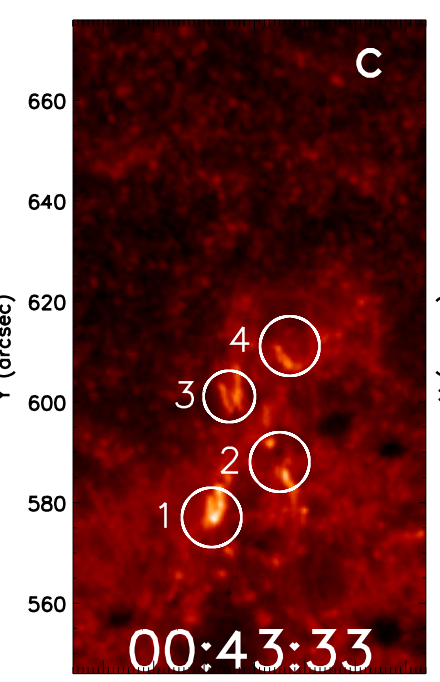

-250-240-230-220-210-200-190 $X$ (arcsec)

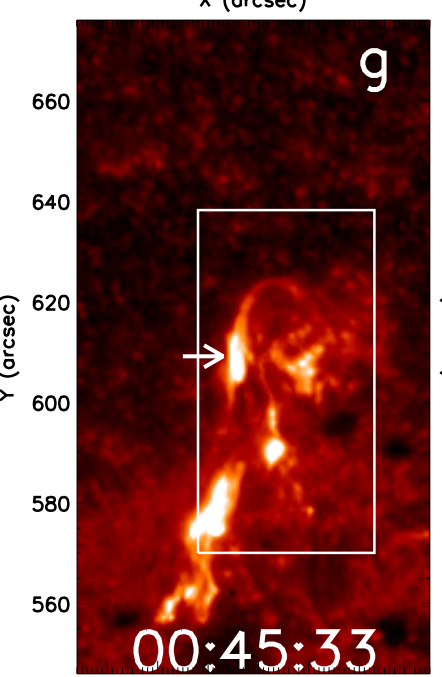

$-250-240-230-220-210-200-190$ $x(\operatorname{arcsec})$

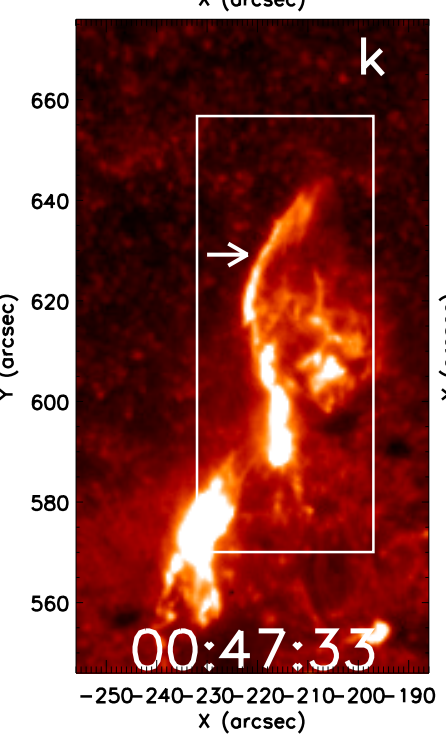

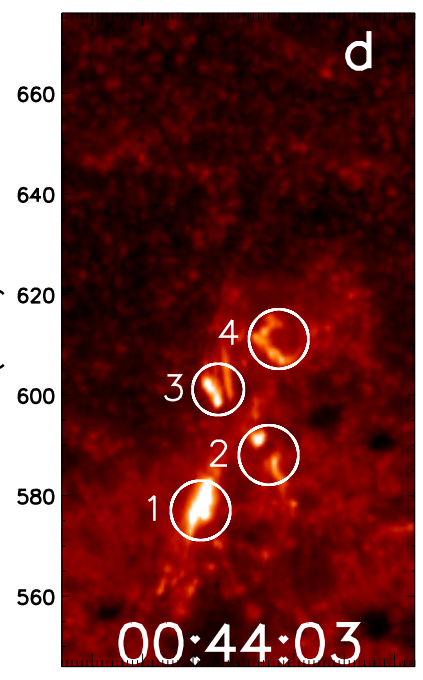

$-250-240-230-220-210-200-190$ $x$ (arcsec)
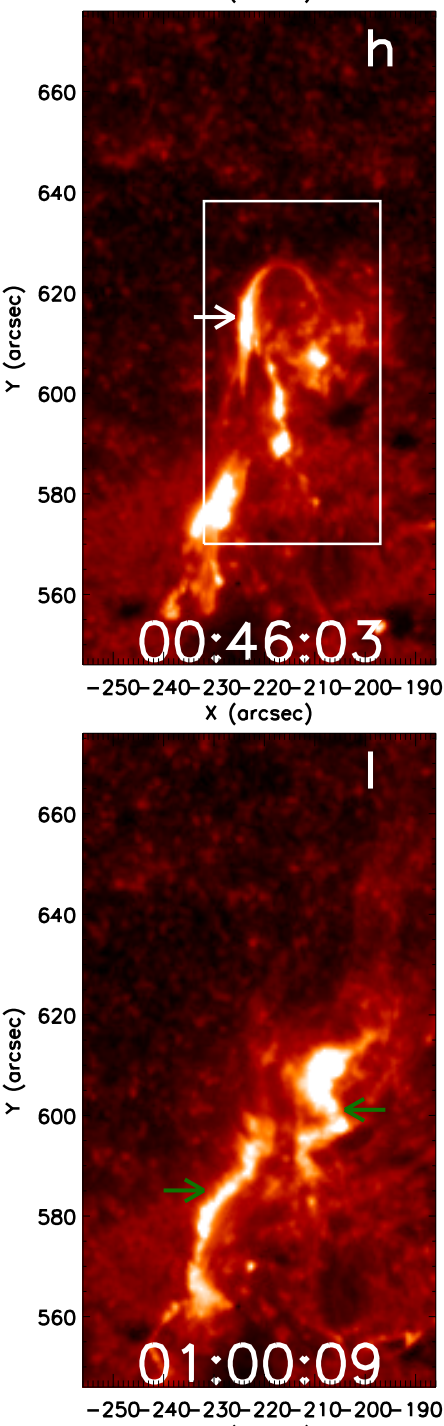

$x$ (arcsec)

Fig. 4. Evolution of the eruptive filament in TRACE $1600 \AA$ A channel from 00:42:33 UT to 01:00:09 UT on 2000 February 9. Panels a)-e) show the onset of the C6.6 flare and the appearance of the bright patches in the chromosphere. Panels f)-k) show the rising of the filament companying the C6.6 flare. The white circles in panels b)-d) indicate the locations of the brigh patches. The white boxes indicate the positions of the filament with time. The white arrows indicate the bright material that was drained by the filament gradually. Two green arrows indicate two flare ribbons. North is upward and west is to the right in all the images in this paper. 

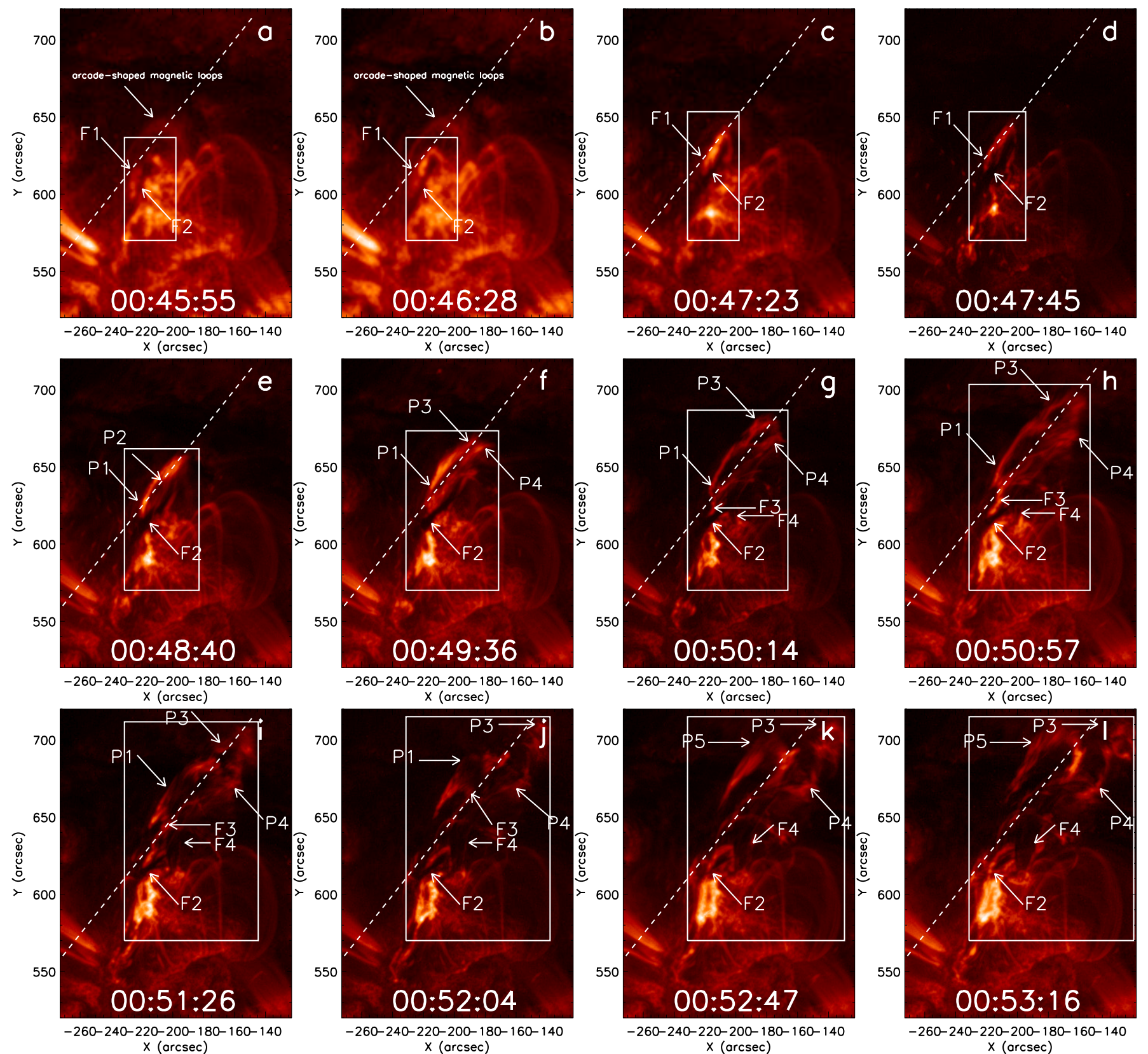

Fig. 5. Evolution of the eruptive filament in TRACE $171 \AA$ A channel from 00:45:55 UT to 00:53:16 UT on 2000 February 9. The filament motion can be seen within the white boxes. The dashed lines show the path along which the filament trajectory is displayed in Fig. 5. The white arrows are described in the text. The position of the white boxes in panels a) and $\mathbf{b}$ ) is the same as those boxes in Figs. $4 \mathrm{f}-4 \mathrm{i}$. The position of the white boxes in panel c) is the same as those boxes in Figs. $4 \mathrm{j}$ and $4 \mathrm{k}$.

crossing points marked by the bright arrows in Figs. 10d-10f were rising from 02:54 UT to 03:54 UT on Feb. 9.

\section{Conclusion and Discussion}

We investigated an active-region filament eruption associated with a C6.6 flare and a CME in NOAA AR 08858 on Feb. 9, 2000 using the GOES 10 soft X-ray flux, BBSO H $\alpha$, SOHO/EIT $304 \AA$ A TRACE $1600 \AA$ and $171 \AA$ images, SOHO/MDI 96-min magnetograms, and SOHO/LASCO C2 images. The main results are as follows: First, the four bright patches first appeared near the filament in the chromosphere before the filament expansion. Three of the four bright patches formed the two ribbons of the flare finally. One bright patch may be the elevated filament material. Second, the intertwined bright and dark strands of the filament expanded first. The bright strands were divided into three parts, which had a different expanding velocity. Next, the erupting filament-carrying flux rope expanded rapidly and then combined with the lower part of the expanding bright strands. The filament erupted, accompanying the other dark strands, which were overlying the filament.

In this event, the appearance of the brightenings in the chromosphere was the precursor of the filament expansion. Consequently, the occurrence of the C6.6 flare resulted in the instability of the filament. Sterling \& Moore (2005) and Sterling et al. (2011) found the appearance of the EUV brightenings before the rising of the filament. X-ray brightenings were also observed before the impulsive phase of the flare and filament acceleration by Chifor et al. (2007). These transient preflare 
X. L. Yan et al.: Case study of a complex active-region filament eruption
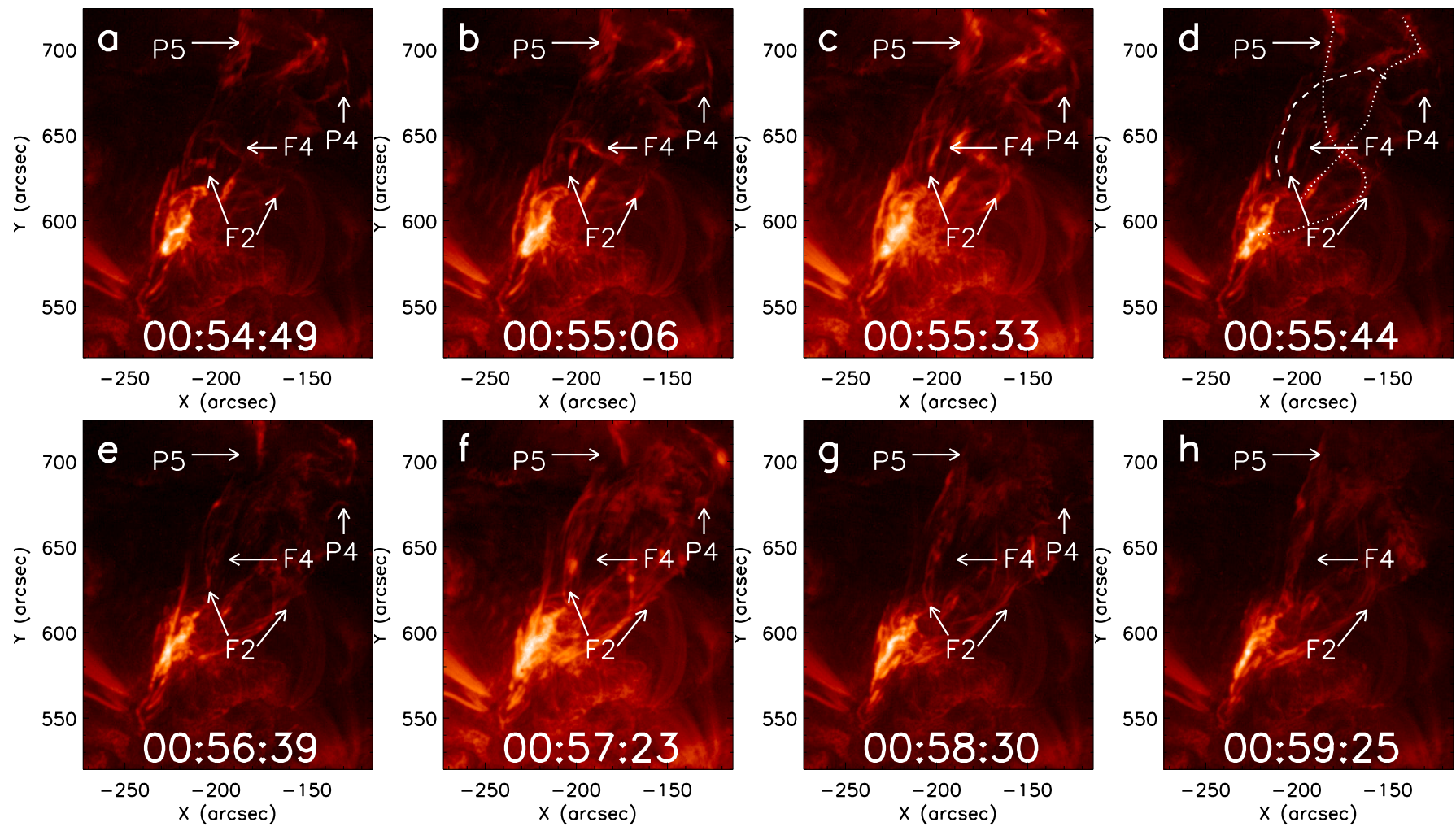

Fig. 6. Evolution of the eruptive filament in TRACE $171 \AA$ channel from 00:54:49 UT to 00:59:25 UT on 2000 February 9. The dotted line indicates the filament in panel d), and the dashed line indicates the dark strands. The white arrows are described in the text.
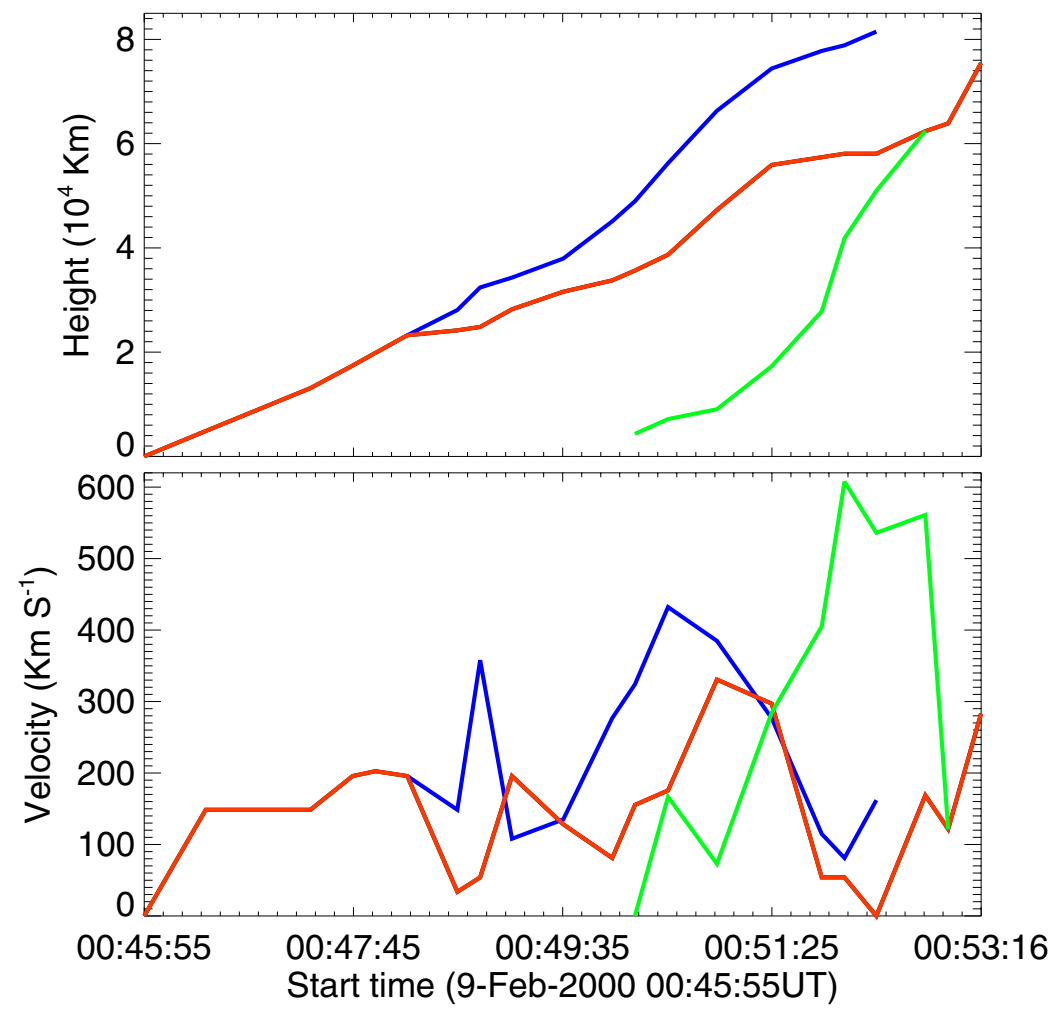

Fig. 7. Height (upper panel) and the velocity (lower panel) of the bright strand (P1) (red line), the bright strand (P3) (blue line), and the main filament (F3) (green line) as a function of time, as measured from $171 \AA$ images along the dashed lines of Fig. 5. We adopted the average values of three repeated measurements of the height. The velocity is the derivative of the height curve.

brightenings were found by Sterling et al. (2011) and Chifor et al. (2007) on or near the polarity inversion line (PIL), coincident with emerging and/or canceling magnetic flux (Schmieder et al. 2008b). Meanwhile, we found that the four bright patches occurred near the PIL. The brightenings could be an indication of tether cutting.
From the change of the projection height and velocity of the filament and the bright strands, the intertwined bright and dark strands of the filament expanded together in the initial phase. Next, the two parts of the bright strands experienced acceleration twice and the main filament-carrying flux rope acceleration followed the two parts of the bright strands acceleration. There 

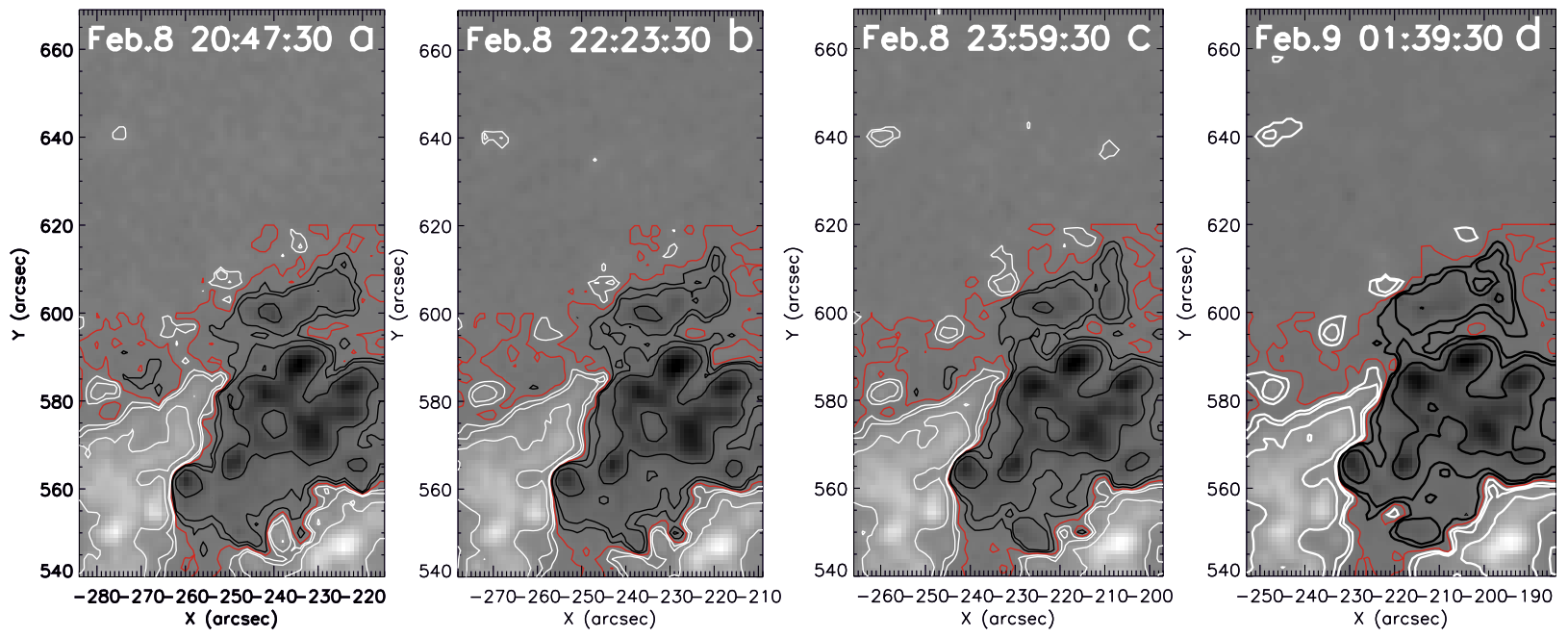

Fig. 8. A sequence of SOHO/MDI magnetograms from 20:47:30 UT on Feb. 8 to 01:39:30 UT on Feb. 9. The white and the black patches denote the positive polarity and the negative polarity, respectively. The levels of the contours of the magnetic fiels are $\pm 50 \mathrm{G}, \pm 100 \mathrm{G}$, and $\pm 300 \mathrm{G}$. The red lines denote the polarity inversion lines.
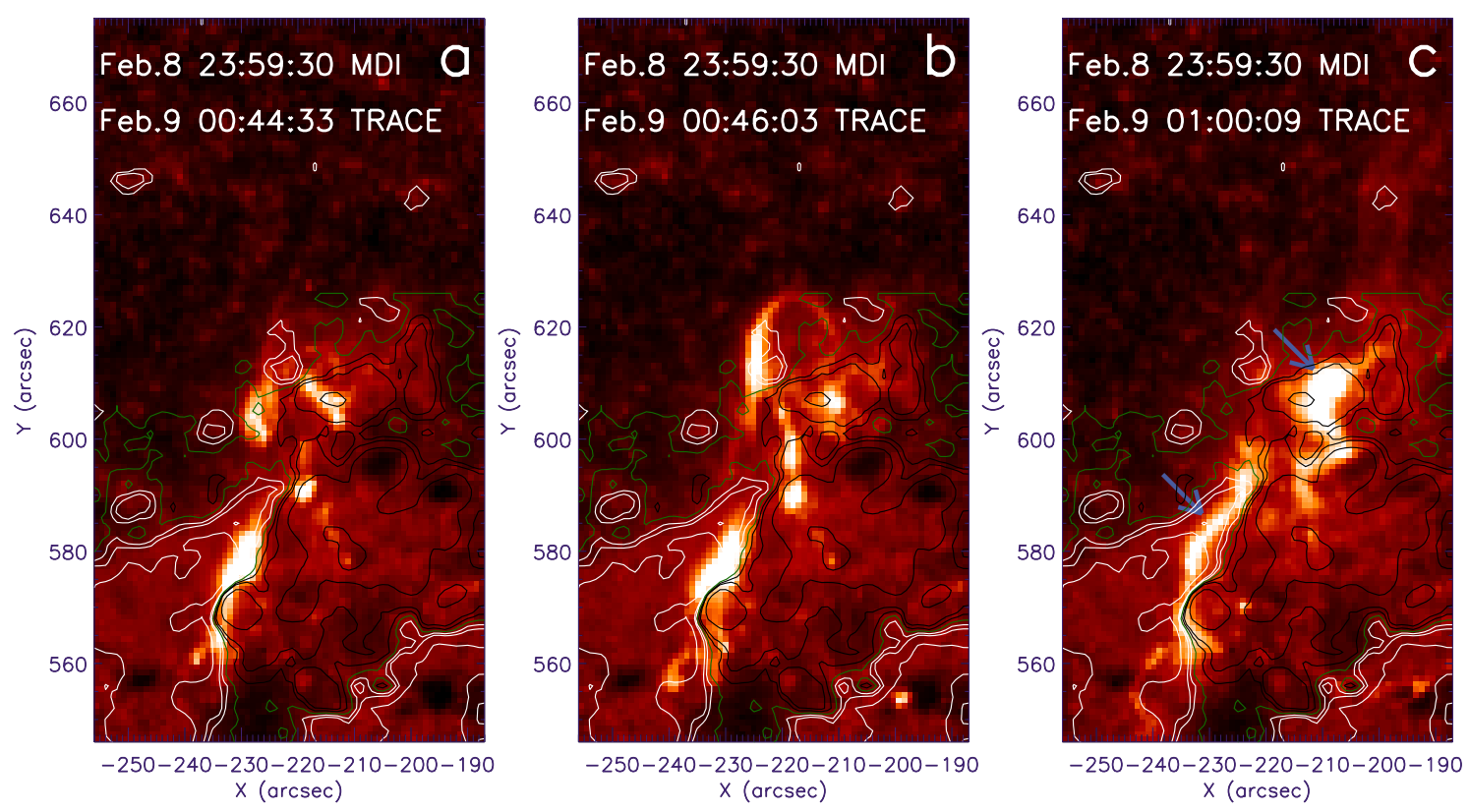

Fig. 9. A sequence of TRACE $1600 \AA$ images with the SOHO/MDI magnetograms at 23:59:30 UT on Feb. 8 superimposed. The solid white and the black lines are the contours of the positive and the negative magnetic fields, respectively. The levels of the contours of the magnetic fields are $\pm 50 \mathrm{G}, \pm 100 \mathrm{G}$, and $\pm 300 \mathrm{G}$. The green lines denote the polarity inversion lines. Two blue arrows indicate the two flare ribbons.

is a time delay between the velocity peaks of two parts of the bright strands and that of the main filament in the velocity time curves. However, the time delay had not been found in the filament observations and simulations of Schrijver et al. (2008).

The previous observations of filament eruptions often demonstrate that filaments often undergo relatively slow rising motions shortly before the onset of solar eruptions (Sterling \& Moore 2005; Chifor etal. 2007; Schmieder et al. 2008a). Nevertheless, the change of the bright strand height with time in this event was very smooth, and the main filament rose rapidly until its eruption. Archontis \& Török (2008) suggested that the mechanism responsible for the fast rise and eruption of the rope is the effective reconnection process that occurs beneath the flux rope and removes the ambient field above it. In this event, the expansion of the two parts of the bright strands resulted in the rapid acceleration of the main filament. Fan (2005) performed MHD simulations to show the evolution of the threedimensional coronal magnetic field as a twisted magnetic flux tube that emerges slowly into the corona. They found that the flux rope was found to undergo kink motion and erupted through the arcade field at a localized region with most of the arcade field remaining closed when the twist in the emerged flux rope reaches some critical level. The simulations of Roussev et al. (2003) and Antiochos et al. (1999) and the observation of Shen et al. (2011) have shown that the presence of the overlying arcade fields can prevent the escape of the twisted loop. The time delay between the velocity peaks of the filament and that of the two parts of the bright strands clearly demonstrates that the effect of the strands of the filament is very important for filament eruption. Since the two ribbons of the flare appeared in the final phase of the filament eruption, it reveals that the rapid magnetic reconnection occurred in the final phase of the filament eruption. 

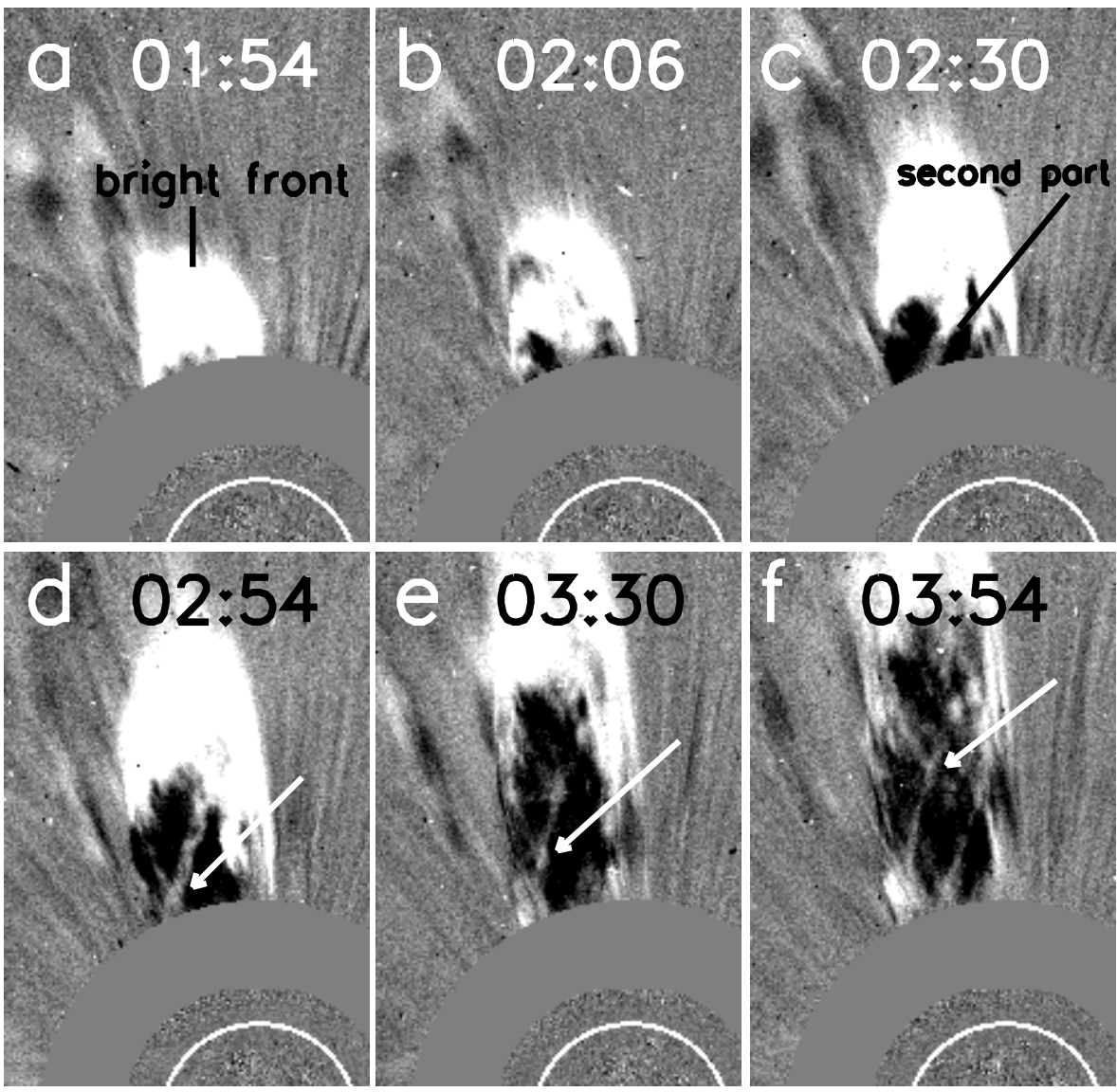

Fig. 10. Running difference image observed by the SOHO/LASCO on Feb. 9 after the filament eruption. The white arrows denote the crossing position of the twisted structure with time. The bright front and the second bright part of CME are all marked in the figure. The field-of-view is $3570^{\prime \prime} \times 5236^{\prime \prime}$.

Before the filament eruption, we had not found the magnetic flux emergence and cancellation. The magnetic field lines over the filament were not observed for reconstruction before the filament eruption. The system of the filament consisted of the bright and the dark strands. The time delay between the velocity peaks of the filament and that of the two parts of the bright strands clearly demonstrates that the breakup of the filament into individual strands is important for its eruption. According to the observation, the eruption models (1), (3), and (5) listed in the introduction can be ruled out. Before the filament eruption, the four brightening patches appeared near the filament. The two flare ribbons were formed after the filament eruption. This scenario is very consistent with the tether-cutting model (Moore et al. 2001). During the rising of the filament, the deformation and the top rotation of the filament was observed. These phenomena are consistent with the occurrence of kink instability, but the twist of the flux rope cannot be determined from observation. We cannot conclude whether the kink instability occurred. Consequently, our observation supports that the tether-cutting of magnetic loops occurred before the filament eruption. The coronal loops may be composed of many strands (Peter et al. 2013). In this event, we observed that the bright loop split into several strands. Meanwhile, we have shown the importance of the breakup of the bright loops that tie on the filament into individual strands for the filament eruption. In this event, the expansion of the bright strands tying on the filament occurred before the filament eruption. With that in mind, the magnetic field line around the filament gives the filament a binding force, which prevents the filament eruption. When these magnetic field lines are removed or expanded, the filament becomes instable and begins to erupt. The filament system studied in this paper has several parts of magnetic field structures. These magnetic structures are coupled together. By analyzing this event, we emphasize the importance of the strands of the filament for filament eruption. This issue needs to be further investigated through simulations. The process of the filament eruption is a multiple process that is physically coupled together rather than a single process.

Acknowledgements. The authors would like to thank the referee for constructive suggestions. The authors thank the TRACE, SOHO, BBSO, and GOES consortia for their data. SOHO is a project of international cooperation between ESA and NASA. This work is supported by the National Science Foundation of China (NSFC) under grant numbers 11373066, 11078005 and 40636031, Key Laboratory of Solar Activity of CAS under number KLSA201303, and the National Basic Research Program of China 973 under grant number G2011CB811400

\section{References}

Alexander, D., Liu, R., \& Gilbert, H. R. 2006, ApJ, 653, 719

Amari, T., Luciani, J. F., Mikic, Z., \& Linker, J. 1999, ApJ, 518, 57

Antiochos, S. K., DeVore, C. R., \& Klimchuk, J. A. 1999, ApJ, 510, 485

Archontis, V., \& Török, T. 2008, A\&A, 492, L35

Aulanier, G., Török, T., Démoulin, P., \& DeLuca, E. E. 2010, ApJ, 708, 314

Brueckner, G. E., Howard, R. A., Koomen, M. J., et al. 1995, Sol. Phys., 162 357

Chen, P. F., \& Shibata, K. 2000, ApJ, 545, 524

Chifor, C., Tripathi, D., Mason, H. E., \& Dennis, B. R. 2007, A\&A, 472, 967

Delaboudinière, J. P., Artzner, G. E., Brunaud, J., et al. 1995, Sol. Phys., 162, 291

Domingo, V., Fleck, B., \& Poland, A. I. 1995, Sol. Phys., 162, 1 
DeVore, C. R., \& Antiochos, S. K. 2008, ApJ, 680, 740

Gaizauskas, V. 1998, in New Perspectives on Solar Prominences, IAU Colloq

167, eds. D. Webb, D. Rust, \& B. Schmieder, ASP Conf. Ser., 150

Gibson, S. E., \& Fan, Y. 2006, J. Geophys. Res., 111, 12103

Forbes, T. G. 1990, J. Geophys. Res., 95, 11919

Forbes, T. G. 2000, J. Geophys. Res., 105, 23153

Forbes, T. G., \& Isenberg, P. A. 1991, ApJ, 373, 294

Feynman, J., \& Martin, S. F. 1995, J. Geophys. Res., 100, 3355

Fan, Y. 2005, ApJ, 630, 543

Gary, G. A., \& Moore, R. L. 2004, ApJ, 611, 545

Handy, B. N., Acton, L. W., Kankelborg, C. C., et al. 1999, Sol. Phys., 187, 229

Hood, A. W., \& Priest, E. R. 1979, Sol. Phys., 64, 303

Ji, H., Wang, H., Schmahl, E, J., Moon, Y. J., \& Jiang, Y. 2003, ApJ, 595, L135

Jing, J., Yurchyshyn, V. B., Yang, G., Xu, Y., \& Wang, H. 2004, ApJ, 614, 1054

Jiang, Y. C., Shen, Y. D., \& Wang, J. X. 2007, Chin. J. Astron. Astrophys., 7, 129

Moore, R. L., \& LaBonte, B. J. 1980, In Solar and Interplanetary Dynamics, eds

M. Dryer, \& E. Tandberg-Hanssen (Boston: Reidel), IAU Symp., 91, 207

Moore, R. L., Sterling, A. C., Hudson, H. S., \& Lemen, J. R. 2001, ApJ, 552, 833

Martin, S. F. 1998, Sol. Phys., 182, 107

Lin, J., \& Forbes, T. G. 2000, J. Geophys. Res., 105, 2375

Lin, J., Forbes, T. G., Isenberg, P. A., \& Demoulin, P. 1998, ApJ, 504, 1006

Lin, J., Forbes, T. G., \& Isenberg, P. A. 2001, J. Geophys. Res., 106, 25053

Linker, J. A., Mikic, Z., Lionello, R., et al. 2003, Phys. Plasmas, 10, 5

Lynch, B. J., Antiochos, S. K., MacNeice, P. J., Zurbuchen, T. H., \& Fisk, L. A. 2004, ApJ, 617, 589

Priest, E. R., \& Forbes, T. G. 2002, A\&ARv, 10, 313

Priest, E. R., Hood, A. W., \& Anzer, U. 1989, ApJ, 344, 1010
Peter, H., Bingert, S., Klimchuk, J. A., et al. 2013, A\&A, 556, A104

Rust, D. M., \& Kumar, A. 1994, Sol. Phys., 155, 69

Rust, D. M., \& Labonte, B. J. 2005, ApJ, 622, L69

Roussev, I. I., Forbes, T. G., Gombosi, T. I., et al. 2003, ApJ, 588, L45

Shibata, K., Masuda, S., Shimojo, M., et al. 1995, ApJ, 451, L83

Steinegger, M., Denker, C., Goode, P. R., et al. 2000, in The Solar Cycle and Terrestrial Climate, ed. A. Wilson, ESA, SP, 463, 617

Scherrer, P. H., Bogart, R. S., Bush, R. I., et al. 1995, Sol. Phys., 162, 169

Schmieder, B., Bommier, V., Kitai, R., et al. 2008a, Sol. Phys., 247, 321

Schmieder, B., Török, T., \& Aulanier, G. 2008b, in Exploring the Solar System and the Universe, eds. V. Mioc, C. Dumitrche, \& N. A. Popescu (Melville, NY: AIP), AIP Conf. Proc., 1043, 260

Schrijver, C. J., Elmore, C., Kliem, B., Török, T., \& Title, A. M. 2008, ApJ, 674, 586

Sterling, A. C., \& Moore, R. L. 2005, ApJ, 630, 1148

Sterling, A. C., Moore, R. L., \& Freeland, S. L. 2011, ApJ, 731, 3

Srivastava, A. K., Zaqarashvili, T. V., Kumar, P., \& Khodachenko, M. L. 2010, ApJ, 715, 292

Shen, Y. D., Liu, Y., \& Liu, R. 2011, RAA, 11, 594

Shen, Y. D., Liu, Y., \& Su, J. 2012, ApJ, 750, 12

Titov, V. S., \& Démoulin, P. 1999, A\&A, 351, 707

Török, T., \& Kliem, B. 2005, ApJ, 630, 97

Török, T., Kliem, B., \& Titov, V. S. 2004, A\&A, 413, L27

Williams, D. R., Török, T., Démoulin, P., van Driel-Gesztelyi, L., \& Kliem, B. 2005, ApJ, 628, L163

Wang, Y. M., \& Sheeley, N. R., Jr. 1999, ApJ, 510, L517

van Ballegooijen, A. A., \& Martens, P. C. H. 1989, ApJ, 343, 971

Yan, X. L., Qu, Z. Q., \& Kong, D. F. 2011, MNRAS, 414, 2803

Yan, X. L., Qu, Z. Q., \& Kong, D. F. 2012, AJ, 143, 56

Zhou, G. P., Wang, J. X., Zhang, J., et al. 2006, ApJ, 662, 35 